\title{
Consistent effects of non-invasive vagus nerve stimulation (nVNS) for the acute treatment of migraine: additional findings from the randomized, sham-controlled, double-blind PRESTO trial
}

Paolo Martelletti ${ }^{1}$, Piero Barbanti ${ }^{2}$, Licia Grazzi ${ }^{3}$, Giulia Pierangeli ${ }^{4}$, Innocenzo Rainero ${ }^{5}$, Pierangelo Geppetti ${ }^{6}$, Anna Ambrosini ${ }^{7}$, Paola Sarchielli ${ }^{8}$, Cristina Tassorelli ${ }^{9,10}$, Eric Liebler ${ }^{11^{*}}$, Marina de Tommaso ${ }^{12}$

and on Behalf of the PRESTO Study Group

\begin{abstract}
Background: Non-invasive vagus nerve stimulation (nVNS) has been shown to be practical, safe, and well tolerated for treating primary headache disorders. The recent multicenter, randomized, double-blind, sham-controlled PRESTO trial provided Class I evidence that for patients with episodic migraine, nVNS significantly increases the probability of having mild pain or being pain-free $2 \mathrm{~h}$ post stimulation. We report additional pre-defined secondary and other end points from PRESTO that demonstrate the consistency and durability of nVNS efficacy across a broad range of outcomes.
\end{abstract}

Methods: After a 4-week observation period, 248 patients with episodic migraine with/without aura were randomly assigned to acute treatment of migraine attacks with nVNS $(n=122)$ or a sham device $(n=126)$ during a doubleblind period lasting 4 weeks (or until the patient had treated 5 attacks). All patients received nVNS therapy during the subsequent 4-week/5-attack open-label period.

Results: The intent-to-treat population consisted of 243 patients. The nVNS group $(n=120)$ had a significantly greater percentage of attacks treated during the double-blind period that were pain-free at $60(P=0.005)$ and 120 min $(P=0.026)$ than the sham group $(n=123)$ did. Similar results were seen for attacks with pain relief at $60(P=0.025)$ and $120 \mathrm{~min}(P=0.018)$. For the first attack and all attacks, the nVNS group had significantly greater decreases (vs sham) in pain score from baseline to $60 \mathrm{~min}(P=0.029)$; the decrease was also significantly greater for nVNS at $120 \mathrm{~min}$ for the first attack $(P=0.011)$. Results during the open-label period were consistent with those of the nVNS group during the double-blind period. The incidence of adverse events (AEs) and adverse device effects was low across all study periods, and no serious AEs occurred.

Conclusions: These results further demonstrate that nVNS is an effective and reliable acute treatment for multiple migraine attacks, which can be used safely while preserving the patient's option to use traditional acute medications as rescue therapy, possibly decreasing the risk of medication overuse. Together with its practicality and optimal tolerability profile, these findings suggest nVNS has value as a front-line option for acute treatment of migraine.

Trial registration: ClinicalTrials.gov identifier: NCT02686034.

Keywords: Neuromodulation, Vagus nerve stimulation, Migraine, Pain intensity, Double-blind, Open-label

\footnotetext{
* Correspondence: eric.liebler@electrocore.com

${ }^{11}$ electroCore, Inc., Basking Ridge, NJ, USA

Full list of author information is available at the end of the article
}

(c) The Author(s). 2018, corrected publication November/2018. Open Access This article is distributed under the terms of the Creative Commons Attribution 4.0 International License (http://creativecommons.org/licenses/by/4.0/), which permits

unrestricted use, distribution, and reproduction in any medium, provided you give appropriate credit to the original author(s) and the source, provide a link to the Creative Commons license, and indicate if changes were made. 


\section{Background}

Standard pharmacologic agents for the acute treatment of migraine can be limited by side effects, inconsistent efficacy, contraindications, risk of drug interactions, and their potential contribution to migraine chronification and medication overuse headache [1-5]. Opioids should be discouraged for the acute treatment of migraine due to significant safety concerns and lack of documented efficacy but remain frequently used in the emergency department setting, which significantly increases healthcare costs [6-9]. Practical alternatives are needed to address this healthcare challenge. Non-invasive neuromodulation therapies could represent a novel option for these patients $[10,11]$.

Non-invasive vagus nerve stimulation (nVNS; gammaCore ${ }^{\oplus}$; electroCore, Inc., Basking Ridge, NJ, USA) demonstrated efficacy in studies of acute migraine treatment and has a strong safety and tolerability profile [12-15]. The multicenter, randomized, double-blind, sham-controlled PRESTO trial provided Class I evidence that for patients with an episodic migraine, acute treatment of migraine attacks with nVNS significantly increases the probability of having mild pain or being pain-free $2 \mathrm{~h}$ post stimulation [11]. The study also clearly demonstrated the practicality, safety, and tolerability of $n V N S$. Here, we report additional pre-defined secondary and other end points from the PRESTO study to illustrate the consistency and durability of nVNS effects across a broad range of outcomes.

\section{Methods}

\section{Study design}

Complete details of the methodology of the multicenter, randomized, double-blind, sham-controlled PRESTO trial have been reported previously [11]. The study was conducted across 10 Italian sites from January 11, 2016, through March 31, 2017, and consisted of an observational period, double-blind period, and open-label period (Fig. 1a). During the observational period, patients treated their migraine attacks with standard medications according to their individual prescriptions. Patients subsequently treated up to 5 migraine attacks with nVNS or sham stimulation during the double-blind period and up to 5 additional attacks with nVNS during the open-label period; only 1 attack could be treated in a 48-h period.

\section{Patients}

Study patients were 18 to 75 years of age, had a previous diagnosis of migraine with or without aura according to the International Classification of Headache Disorders, 3rd edition (beta version) criteria [16], were $<50$ years of age at migraine onset, and had 3 to 8 migraine attacks per month with $<15$ headache days per month during the last 6 months. Patients who were receiving preventive migraine medications at baseline (or other preventive medications determined to potentially interfere with the study) were required to have maintained a stable dose and frequency of these medications during the 2 months

a

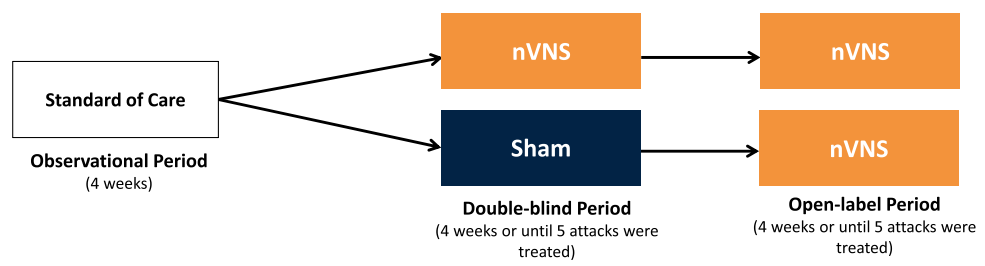

b

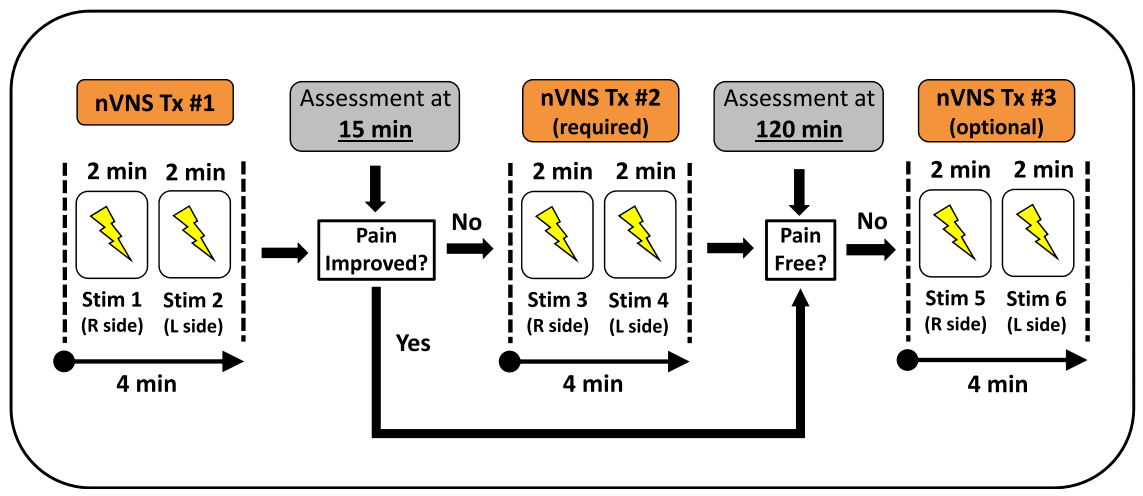

Fig. 1 PRESTO study design (a) and treatment protocol (b). Abbreviations: L, left; nVNS, non-invasive vagus nerve stimulation; R, right; Stim, stimulation; TX, treatment 
before enrollment and throughout the study; initiation of new preventive medications was not permitted during this period.

\section{Interventions and study procedures}

Patients were randomly assigned (1:1) to receive nVNS or sham (variable block design $[4,6]$, stratified by site). Full details of study randomization and blinding, as well as information about the active and sham devices, have been described previously [11].

Within $20 \mathrm{~min}$ of migraine pain onset, patients self-administered bilateral 120-s stimulations (ie, 1 stimulation each to the right and left sides of the neck) (Fig. 1b) and recorded post-treatment assessments in their study diaries $15,30,60$, and $120 \mathrm{~min}$ and 24 and $48 \mathrm{~h}$ after completion of the initial bilateral stimulations. Patients were instructed to repeat the bilateral stimulations if pain had not improved at the 15-min assessment, and those who were not pain-free at the 120-min assessment had the option of administering an additional set of bilateral stimulations. Patients were asked to wait $120 \mathrm{~min}$ from the first set of stimulations before using acute rescue medication.

\section{End points}

Here, we present clinically relevant secondary and other end points not included in the original PRESTO publication to provide a more comprehensive depiction of the data set. Pain-free was defined as a score of 0 on the 4-point headache pain scale (with 0 indicating no pain and 3 indicating severe pain), and pain relief was defined as a score of 0 or 1 (both without use of rescue medication before $120 \mathrm{~min}$ ) in a subject with pain of at least moderate severity at baseline. Attacks with mild pain (ie, a pain score of 1) at both baseline and the subsequent time point of interest were considered treatment failures. These definitions were used in assessment of the following end points:

- Percentages of all treated attacks that achieved pain freedom and pain relief at 30, 60, and $120 \mathrm{~min}$ for the double-blind period and at $120 \mathrm{~min}$ for the open-label period;

- Mean change in pain score from baseline to 30, 60, and $120 \mathrm{~min}$ for the first attack and for all attacks in the double-blind and open-label periods;

- Number of acute medications used per migraine attack during the observational, double-blind, and open-label periods;

- Sustained treatment response (defined as pain-free or pain relief [without use of rescue medication] at both $2 \mathrm{~h}$ and $24 \mathrm{~h}$ or at 2, 24, and $48 \mathrm{~h}$ ) rates for the first attack and all attacks for the double-blind and open-label periods;

- Incidence of adverse events (AEs) and adverse device effects (ADEs).

\section{Statistical methods}

All efficacy end points were evaluated using the intentto-treat (ITT) population, defined as all randomly assigned patients who treated at least 1 migraine attack in the double-blind period. Descriptive statistics were used to summarize continuous variables (means and $95 \%$ confidence intervals [CIs]) and categorical variables (frequency counts, percentages, and 95\% CIs). Generalized linear mixed effects regression models were used to estimate the proportion of all attacks that were pain-free or had pain relief for the nVNS and sham groups, allowing for both subject-specific and populationaveraged inferences in non-normally distributed data; $P$ values were from resulting $\mathrm{F}$ tests. Mean change from baseline pain score was compared between treatment groups via 2-sample $t$ tests for the first attack and via linear mixed effects regression models. Poisson regression was used to compare medication use per attack between treatment groups. For sustained treatment response (pain-free and pain relief), the nVNS and sham groups were compared via the chi-square test or Fisher exact test, as appropriate, for the first attack and via linear mixed effects regression models for all attacks. All data were analyzed using $\mathrm{SAS}^{\circ}$ 9.4 (SAS Institute Inc., Cary, NC, USA). Two-sided $P$ values $<0.05$ were considered statistically significant.

\section{Results}

Patients

Complete descriptions of patient disposition, demographics, and baseline characteristics in PRESTO are included in the original study publication [11]. The ITT population consisted of 243 patients (nVNS, $n=$ 120; sham, $n=123)$. Two hundred thirty-nine patients entered the open-label period ( $\mathrm{VVNS}, n=117$; sham, $n=122)$; among these, 238 patients $(>99 \%)$ completed this period (1 patient was lost to follow-up), with 220 (92\%) treating at least 1 attack during the period. One patient who treated at least 1 attack in and completed the open-label period was not part of the ITT population. Table 1 summarizes key demographics and other key patient characteristics.

\section{Efficacy: double-blind period}

In the nVNS group, the percentage of all attacks that were pain-free at $60 \mathrm{~min}(16.3 \%)$ and $120 \mathrm{~min}(22.9 \%)$ was significantly greater than in the sham group $(8.6 \%$ and $14.8 \%$, respectively; $P<0.05$ for both time points) (Fig. 2a). Similar significant results were seen for the 
Table 1 Demographics and other key patient characteristics

\begin{tabular}{|c|c|c|c|}
\hline Characteristic & nVNS $(n=120)$ & Sham $(n=123)$ & Total $(N=243)$ \\
\hline Age, mean (SD), y & $38.8(11.0)$ & $39.6(11.8)$ & $39.2(11.4)$ \\
\hline Female sex, No. (\%) & $95(79.2)$ & $91(74.0)$ & $186(76.5)$ \\
\hline \multicolumn{4}{|l|}{ Diagnosis, No. (\%) } \\
\hline Migraine with aura & $8(6.7)$ & $9(7.3)$ & $17(7.0)$ \\
\hline Migraine without aura & $112(93.3)$ & $114(92.7)$ & $226(93.0)$ \\
\hline $\begin{array}{l}\text { Current preventive medication } \\
\text { use, No. (\%) }\end{array}$ & $42(35.0)$ & $35(28.5)$ & $77(31.7)$ \\
\hline $\begin{array}{l}\text { No. of acute medication days } \\
\text { per mo, }{ }^{a} \text { mean (SD) }\end{array}$ & $5.6(1.7)$ & $5.3(1.7)$ & $5.5(1.7)$ \\
\hline $\begin{array}{l}\text { Attack severity at onset for all treated } \\
\text { attacks in DB period, No. (\%) }\end{array}$ & $n=359^{\mathrm{b}}$ & $n=329^{b}$ & NA \\
\hline Mild & $113(31.5)$ & $105(31.9)$ & \\
\hline Moderate & $156(43.5)$ & $166(50.5)$ & \\
\hline Severe & $90(25.1)$ & $58(17.6)$ & \\
\hline $\begin{array}{l}\text { Attack severity at onset for first } \\
\text { treated attack in DB period, No. (\%) }\end{array}$ & $n=119^{b}$ & $n=119^{b}$ & NA \\
\hline Mild & $40(33.6)$ & $46(38.7)$ & \\
\hline Moderate & $51(42.9)$ & $55(46.2)$ & \\
\hline Severe & $28(23.5)$ & $18(15.1)$ & \\
\hline
\end{tabular}

Tassorelli C, Grazzi L, de Tommaso M, et al. Non-invasive vagus nerve stimulation as acute therapy for migraine: the randomized PRESTO study. Neurology. 2018;91(4):e364-e373

Abbreviations: $D B$ Double-blind, NA Not applicable, $n V N S$ Non-invasive vagus nerve stimulation, SD Standard deviation

${ }^{a}$ No. of days the patient typically takes acute migraine medication per month.

${ }^{b}$ Patients with no reported baseline severity were excluded from this analysis

percentage of attacks with pain relief (Fig. 2b), which was $29.4 \%$ for the nVNS group and $20.3 \%$ for the sham group at $60 \min (P=0.025)$ and $35.2 \%$ for the nVNS group and $25.4 \%$ for the sham group at $120 \min (P=0.018)$.

For the first attack (Fig. 3a), the nVNS group had significantly greater decreases (vs the sham group) in mean pain score from baseline at $60 \mathrm{~min}$ (nVNS, 0.51; sham, $0.22 ; P=0.029$ ) and $120 \mathrm{~min}$ (nVNS, 0.62; sham, 0.23; $P=0.011$ ). For all attacks (Fig. 3c), the mean decrease from baseline in pain score was significantly greater in the nVNS group (0.42) than in the sham group (0.22) at $60 \mathrm{~min}(P=0.029)$ but not at $120 \mathrm{~min}$ (nVNS, 0.50; sham, 0.28; $P=0.057$ ).

During the observational (run-in) period, study patients used a mean of 0.86 acute medications per attack. Acute medication use decreased during the double-blind period to 0.45 medications per attack in the nVNS group and 0.55 medications per attack in the sham group $(P=0.055)$.

Sustained pain-free and pain relief response rates were high in both the nVNS and sham groups at $24 \mathrm{~h}(\geq 75 \%)$ and $48 \mathrm{~h}(\geq 58 \%)$ for both the first attack and all attacks (Table 2).

\section{Efficacy: open-label period}

The percentages of all treated attacks that were pain-free (23.3\%) or had pain relief $(38.1 \%)$ at 120 min during the open-label period were similar to those of the nVNS group during the double-blind period (Fig. 2). Mean changes from baseline in pain score during the open-label period for the first attack (30 $\mathrm{min},-$ or $\mathrm{t}$; $60 \mathrm{~min},-0.56 ; 120 \mathrm{~min},-0.61)$ and for all treated attacks (30 min, -0.38 ; $60 \mathrm{~min},-0.50 ; 120 \mathrm{~min},-0.57$ ) were similar to those seen in the nVNS group during the double-blind period (Fig. 3). A mean of 0.46 acute medications per attack were used during the open-label period, which was similar to that of the nVNS group during the double-blind period $(0.45$ acute medications per attack) and was decreased from
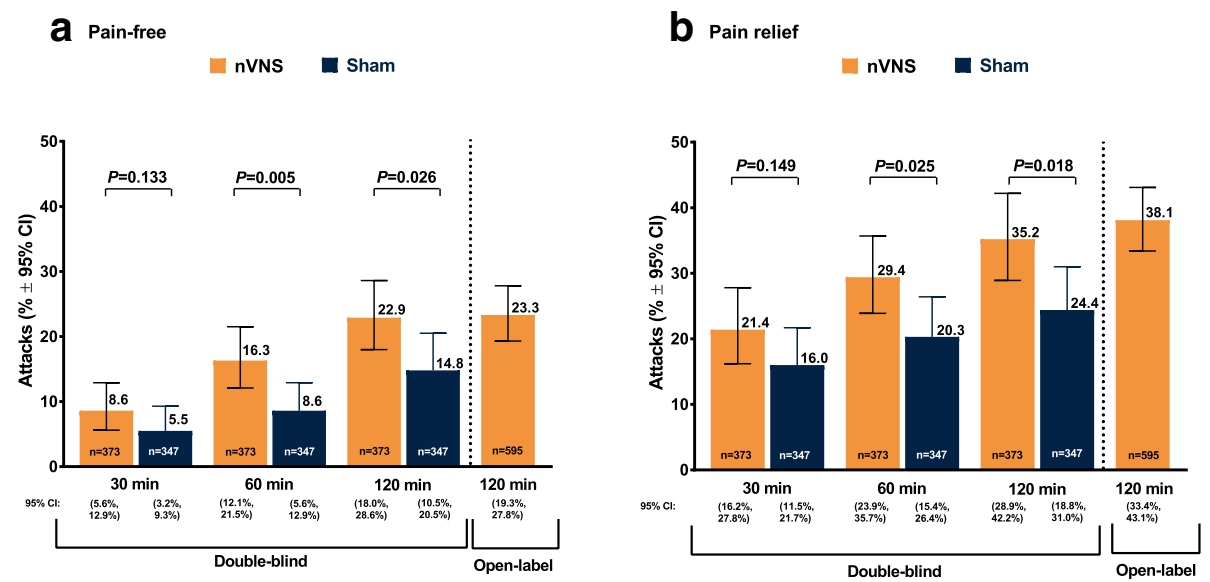

Fig. 2 Percentage of all treated attacks that were pain-free (a) or had pain relief (b) during the double-blind and open-label periods (ITT population, $N=243$ ). Generalized linear mixed effects regression models were used to estimate the proportion of successful responses, allowing for both subjectspecific and population-averaged inferences in non-normally distributed data. $P$ values are from resulting $F$ tests. Models were adjusted for subject's pain score at baseline, use of preventive therapies, and indicator or presence of aura. Abbreviations: Cl, confidence interval; ITT, intent-to-treat; $n V N S$, non-invasive vagus nerve stimulation 


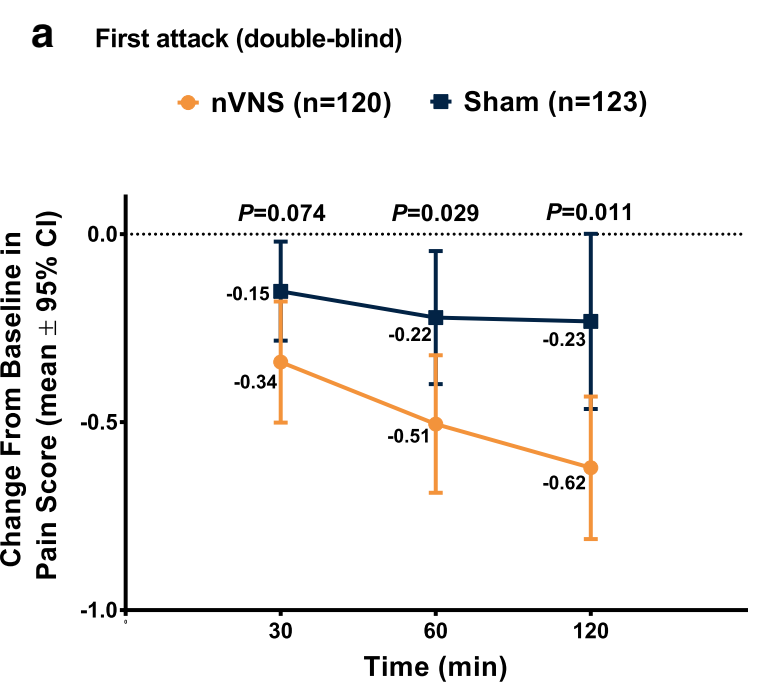

C All attacks (double-blind)

- nVNS $(n=325)$ Sham $(n=300)$

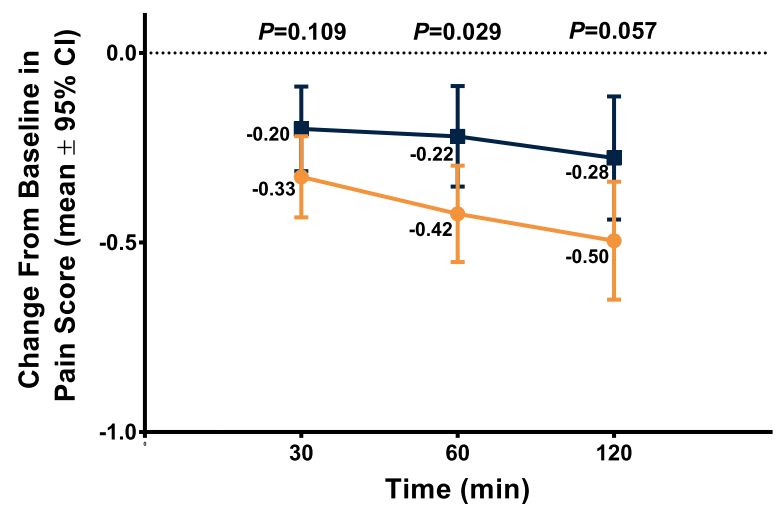

b First attack (open-label)

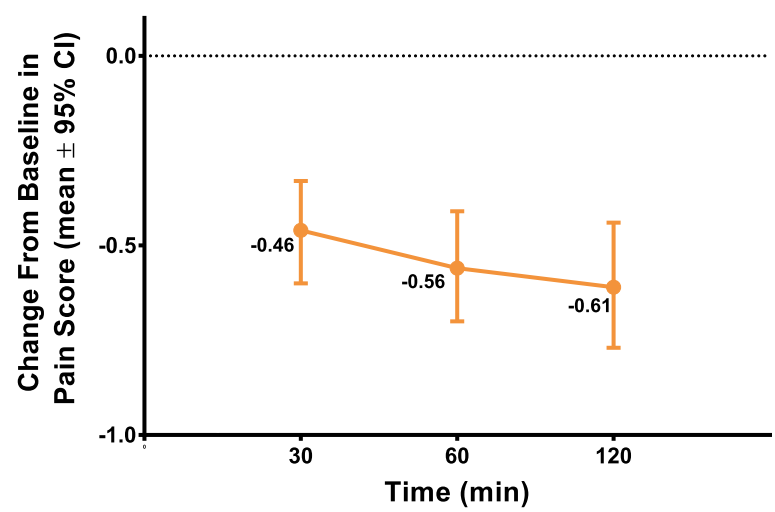

d All attacks (open-label)

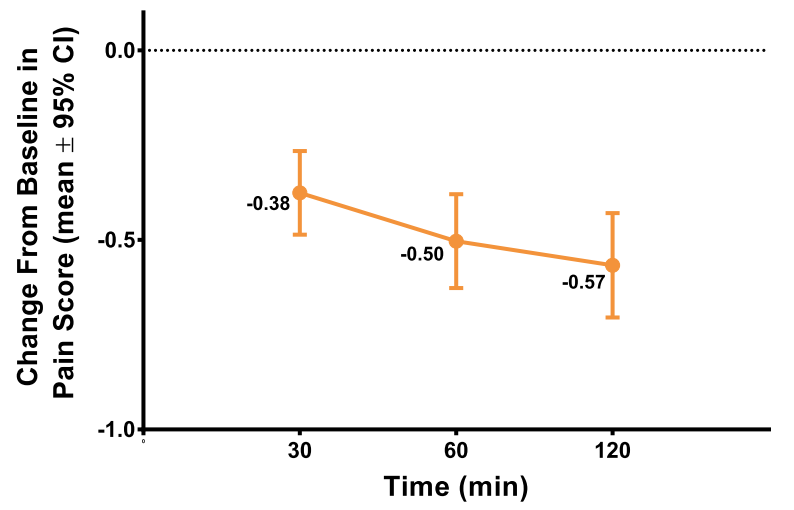

Fig. 3 Mean change in pain score from baseline for first attack during the double-blind (a) and open-label (b) periods and for all attacks during the double-blind (c) and open-label Periods (d). $P$ values for first attack are from 2-sample $t$ tests. For all attacks, linear mixed effects regression models were used to estimate the change in pain score between baseline and 30, 60, and $120 \mathrm{~min}$, allowing for both subject-specific and populationaveraged inferences. Abbreviations: $\mathrm{Cl}$, confidence interval; nVNS, non-invasive vagus nerve stimulation

the observation period by 0.40 acute medications per attack. Sustained treatment response during the open-label period was generally similar to or greater than that seen in the nVNS group during the double-blind period (Table 2).

\section{Safety}

As previously reported [11], the incidence of AEs and ADEs was low across all study periods, and no serious AEs occurred. The only ADE reported by $>1$ patient during the open-label period was vertigo, which was reported by 2 patients (1\%).

\section{Discussion}

These additional results from the PRESTO study further demonstrate that nVNS is superior to sham across a broad range of relevant end points. In the nVNS group, significantly greater percentages of all attacks were pain-free or had pain relief at 60 and $120 \mathrm{~min}$ than in the sham group. The nVNS group also had significantly greater decreases from baseline in mean pain score for the first attack (60 and $120 \mathrm{~min}$ ) and for all attacks (60 min). Among nVNS-treated attacks that were painfree at $120 \mathrm{~min},>75 \%$ had a sustained response at $24 \mathrm{~h}$. Ninety-eight percent of patients in the ITT population completed the open-label period, suggesting that the 
Table 2 Sustained response at 24 and $48 \mathrm{~h}$ post-treatment

\begin{tabular}{|c|c|c|c|c|}
\hline & \multicolumn{3}{|c|}{ Double-blind Period } & \multirow{2}{*}{$\begin{array}{l}\text { Open-label Period } \\
\text { nVNS }\end{array}$} \\
\hline & nVNS & Sham & $P$ Value & \\
\hline & $\begin{array}{l}\%(n / N) \\
(95 \% \mathrm{Cl})\end{array}$ & $\begin{array}{l}\%(\mathrm{n} / \mathrm{N}) \\
(95 \% \mathrm{Cl})\end{array}$ & & $\begin{array}{l}\%(\mathrm{n} / \mathrm{N}) \\
(95 \% \mathrm{Cl})\end{array}$ \\
\hline \multicolumn{5}{|l|}{ All Attacks } \\
\hline \multicolumn{5}{|c|}{ Pain-free } \\
\hline $24 \mathrm{~h}$ & $\begin{array}{l}79.0(71 / 93) \\
(67.9,87.1)\end{array}$ & $\begin{array}{l}83.7(54 / 66) \\
(69.9,91.9)\end{array}$ & $0.532^{a}$ & $\begin{array}{l}80.8(118 / 146) \\
(73.5,86.6)\end{array}$ \\
\hline $48 \mathrm{~h}$ & $\begin{array}{l}65.7(59 / 93) \\
(53.3,76.3)\end{array}$ & $\begin{array}{l}71.2(46 / 66) \\
(56.5,82.5)\end{array}$ & $0.537^{\mathrm{a}}$ & $\begin{array}{l}70.3(102 / 146) \\
(61.6,77.7)\end{array}$ \\
\hline \multicolumn{5}{|c|}{ Pain relief } \\
\hline $24 \mathrm{~h}$ & $\begin{array}{l}80.9(109 / 138) \\
(72.3,87.3)\end{array}$ & $\begin{array}{l}80.3(78 / 99) \\
(69.4,87.9)\end{array}$ & $0.916^{\mathrm{a}}$ & $\begin{array}{l}80.5(185 / 230) \\
(74.5,85.3)\end{array}$ \\
\hline $48 \mathrm{~h}$ & $\begin{array}{l}74.1(99 / 138) \\
(64.0,82.1)\end{array}$ & $\begin{array}{l}72.1(70 / 99) \\
(60.7,81.3)\end{array}$ & $0.775^{\mathrm{a}}$ & $\begin{array}{l}71.9(165 / 230) \\
(65.2,77.7)\end{array}$ \\
\hline \multicolumn{5}{|c|}{ First Attack } \\
\hline \multicolumn{5}{|c|}{ Pain-free } \\
\hline $24 \mathrm{~h}$ & $\begin{array}{l}75.0(27 / 36) \\
(57.8,87.9)\end{array}$ & $\begin{array}{l}84.6(22 / 26) \\
(65.1,95.6)\end{array}$ & $0.359^{b}$ & $\begin{array}{l}75.4(46 / 61) \\
(62.7,85.5)\end{array}$ \\
\hline $48 \mathrm{~h}$ & $\begin{array}{l}58.3(21 / 36) \\
(40.8,74.5)\end{array}$ & $\begin{array}{l}69.2(18 / 26) \\
(48.2,85.7)\end{array}$ & $0.381^{b}$ & $\begin{array}{l}65.6(40 / 61) \\
(52.3,77.3)\end{array}$ \\
\hline \multicolumn{5}{|c|}{ Pain relief } \\
\hline $24 \mathrm{~h}$ & $\begin{array}{l}77.3(58 / 75) \\
(66.2,86.2)\end{array}$ & $\begin{array}{l}79.3(46 / 58) \\
(66.7,88.8)\end{array}$ & $0.784^{b}$ & $\begin{array}{l}78.8(108 / 137) \\
(71.0,85.3)\end{array}$ \\
\hline $48 \mathrm{~h}$ & $\begin{array}{l}69.3(52 / 75) \\
(57.6,79.5)\end{array}$ & $\begin{array}{l}71.0(41 / 58) \\
(57.3,81.9)\end{array}$ & $0.866^{b}$ & $\begin{array}{l}70.1(96 / 137) \\
(61.7,77.6)\end{array}$ \\
\hline
\end{tabular}

Abbreviations: $\mathrm{Cl}$ Confidence interval, $n V N S$ Non-invasive vagus nerve stimulation ${ }^{\mathrm{a}} \mathrm{Generalized} \mathrm{linear} \mathrm{mixed} \mathrm{effects} \mathrm{regression} \mathrm{models} \mathrm{were} \mathrm{used} \mathrm{to} \mathrm{estimate} \mathrm{the}$ proportion of successful responses, allowing for both subject-specific and population-averaged inferences in non-normally distributed data. ${ }^{\mathrm{b}}$ From chi-square test or Fisher exact test, as appropriate

benefit from $\mathrm{nVNS}$ was maintained and that nVNS is a durable acute therapy. The results for the total population during the open-label period were generally similar to those seen for the nVNS group during the double-blind period. Throughout the study, the incidence of AEs and ADEs was low, and no serious AEs were reported.

The findings from PRESTO are consistent with those from other clinical studies of acute nVNS use in migraine $[12,13]$. They are supported by several potential mechanisms of action for the acute benefits of vagus nerve stimulation, including inhibition of central excitability through suppression of glutamate release, suppression of acute nociceptive activation of trigeminocervical neurons, and curbing expression of proteins associated with central sensitization of trigeminal neurons [17-19].

The high rates of sustained 24-h pain-free response to nVNS seen in PRESTO (>75\%) stand in contrast to the lower rates reported for oral triptans $(10 \%-30 \%)$ and single-pulse transcranial magnetic stimulation (29\%) [10, 20]. The protocol of the single-pulse transcranial magnetic stimulation study called for a study population restricted to patients with aura and a treatment time that was independent from the onset of pain (ie, within $1 \mathrm{~h}$ after aura onset), making comparison with the nVNS findings challenging [10]. The majority of patients enrolled in the PRESTO study had migraine without aura, and nVNS was delivered promptly after the onset of migraine pain (ie, within $20 \mathrm{~min}$ ) [11]. In the majority of the triptan clinical trials, treatment was not initiated until migraine pain reached a moderate/severe level, partially due to the desire to avoid unnecessary adverse effects [20, 21]. Consistent with findings from PRESTO [22], rates of sustained response appear to be higher with the use of triptans during the early stages of migraine $(34 \%-53 \%)$ than during the later stages $(19 \%-31 \%)[21,23]$. These observations suggest that there are benefits to treating early in the course of migraine attacks-in a sense, intervening before the migraine process is fully activated. High rates of sustained pain-free response in both the nVNS and sham groups in PRESTO suggest that intervention early in the course of migraine might confer benefits, irrespective of the treatment.

Together with findings from multiple previous studies [12-15, 24-26], these results from PRESTO further highlight the clinical utility, practicality, and flexibility of nVNS. Across the double-blind and open-label periods, $\mathrm{nVNS}$ was used to treat $>900$ migraine attacks, with data collected at multiple time points for each attack, demonstrating its consistent efficacy, safety, and tolerability as acute treatment for these attacks. nVNS can be used as monotherapy or in conjunction with other treatments without risk of pharmacologic interactions, offering a clinical versatility that other acute migraine treatments lack. These advantages, along with its convenience and ease of use, make nVNS an appealing and pragmatic option for early, adjunctive, and/or frequent use in the acute treatment of migraine. nVNS could also help minimize the risk of medication overuse associated with traditional acute treatments and reduce the frequency of opioid use for the acute treatment of migraine in the emergency department setting.

This study has a number of limitations. The selection of an appropriate sham device in neuromodulation studies is challenging. In accordance with previous recommendations to ensure maintenance of the study blind [27], the sham device used in PRESTO produced an active signal that could be perceived by the user but was not designed to stimulate the vagus nerve; recent data suggest that the strength of the sham device's signal may have inadvertently activated the vagus nerve and could have inflated the responses to sham treatment across all end points [28]. This phenomenon, which merits further investigation, may have been related to a psychobiological placebo effect but more likely resulted 
from the unanticipated physiologically active signal that may have decreased the difference in therapeutic gain seen between the nVNS and sham groups [11].

During both the double-blind and open-label periods, the mean number of acute medications used per migraine attack was substantially lower than that seen during the observational period. Such a decrease in medication use could be interpreted as evidence of treatment efficacy; however, these results must be interpreted with caution, as patients were encouraged to refrain from using acute medications for $120 \mathrm{~min}$ after stimulation with the study device. This study limitation most likely contributed to decreases in acute medication use in both the nVNS and sham groups during the double-blind period and may partially explain the lack of significance between treatment groups for this end point.

\section{Conclusions}

These results from clinically relevant secondary and other end points of the PRESTO study demonstrate the efficacy and reliability of $\mathrm{nVNS}$ for the acute treatment of migraine. nVNS provided dependable efficacy for the successful treatment of multiple attacks and can be used safely while preserving a patient's option to use additional acute medications as rescue therapy, thus potentially decreasing the risk of medication overuse. Together, these findings highlight the flexibility and practicality of nVNS as a front-line option for acute migraine attacks.

\section{Abbreviations}

ADE: Adverse device effect; AE: Adverse event; Cl: Confidence interval; DB: Doubleblind; ITT: Intent to treat; L: Left; NA: Not applicable; nVNS: Non-invasive vagus nerve stimulation; R: Right; SD: Standard deviation; Stim: Stimulation; Tx: Treatment

\section{Acknowledgments}

Medical writing support was provided by Elizabeth Barton, MS, of MedLogix Communications, LLC, in cooperation with the authors. Statistical analyses were conducted by Candace McClure, PhD, of North American Science Associates Inc. Co-investigators:

The PRESTO Study Group

Coinvestigators are listed by study site: 1. Headache Science Centre, National Neurological Institute C. Mondino Fo undation and University of Pavia: Cristina Tassorelli, MD, PhD (Principal Investigator); Vito Bitetto (Subinvestigator); Roberto De Icco, MD (Subinvestigator); Daniele Martinelli, MD (Subinvestigator); Grazia Sances, MD (Subinvestigator); Monica Bianchi, MD (Research Nurse); 2. Carlo Besta Neurological Institute and Foundation: Licia Grazzi, MD (Principal Investigator); Anna Maria Padovan (Subinvestigator); 3. University of Bari Aldo Moro: Marina de Tommaso, MD, PhD (Principal Investigator); Katia Ricci (Subinvestigator); Eleonora Vecchio, MD, PhD (Subinvestigator); 4. IRCCS Istituto delle Scienze Neurologiche di Bologna: Pietro Cortelli, MD, PhD (Principal Investigator); Sabina Cevoli, MD, PhD (Subinvestigator); Giulia Pierangeli, MD, PhD (Subinvestigator); Rossana Terlizzi, MD (Subinvestigator); 5. Sapienza University of Rome: Paolo Martelletti, MD, PhD (Principal Investigator); Andrea Negro, MD (Subinvestigator); Gabriella Addolorata Chiariello (Research Nurse); 6. University of Turin: Innocenzo Rainero, MD, PhD (Principal Investigator); Paola De Martino, MD, PhD (Subinvestigator); Annalisa Gai, MD (Subinvestigator); Flora Govone, MD (Subinvestigator); Federica Masuzzo, MD (Subinvestigator); Elisa Rubino, MD, PhD (Subinvestigator); Maria Claudia Torrieri, MD (Subinvestigator); Alessandro Vacca, MD (Subinvestigator); 7. University Hospital of Careggi:
Pierangelo Geppetti, MD, PhD (Principal Investigator); Alberto Chiarugi, MD, PhD (Subinvestigator); Francesco De Cesaris (Subinvestigator); Simone Li Puma (Subinvestigator); Chiara Lupi (Subinvestigator); Ilaria Marone (Subinvestigator); 8. IRCCS Neuromed: Anna Ambrosini, MD, PhD (Principal Investigator); Armando Perrotta, MD, PhD (Subinvestigator); 9. Santa Maria della Misericordia Hospital: Paola Sarchielli, MD, PhD (Principal Investigator); Laura Bernetti, MD (Subinvestigator); Ilenia Corbelli, MD, PhD (Subinvestigator); Michele Romoli, MD (Subinvestigator); Simone Simoni, MD (Subinvestigator); Angela Verzina, MD (Subinvestigator); 10. IRCCS San Raffaele Pisana: Piero Barbanti, MD, PhD (Principal Investigator); Cinzia Aurilia, MD (Subinvestigator); Gabriella Egeo, MD, PhD (Subinvestigator); Luisa Fofi, MD (Subinvestigator). electroCore Study Team: Eric Liebler (Senior Vice President, Neurology); Annelie Andersson (Senior Director, Clinical Director); Lia Spitzer (Senior Director, Clinical/Study Manager); Juana Marin, MD (Clinical Advisor, Safety Monitor); Candace McClure, PhD (North American Science Associates Inc., Statistician); Lisa Thackeray, MS (North American Science Associates Inc. Statistician), Maria Giovanna Baldi (Monitor); Daniela Di Maro (Monitor).

\section{Funding}

This study was sponsored by electroCore, Inc.

\section{Availability of data and materials}

Any data not published within this article will be publicly available at ClinicalTrials.gov with the identifier NCT02686034. Individual participant data will not be shared.

\section{Authors' contributions}

$L G, E L$, and $C T$ contributed to the PRESTO study design. All primary investigators were involved in participant recruitment and treatment for the PRESTO study. All authors participated in data collection, interpretation, and validation and had full access to all study data. CT and EL were involved in data analysis. EL drafted and revised the manuscript for content in cooperation with all authors. All authors reviewed, critiqued, and contributed to revision of the manuscript content and provided approval of the final manuscript draft to be submitted to the Journal of Headache and Pain. The principal author, PM, takes responsibility for all aspects of the work and for ensuring that questions related to the accuracy or integrity of any part of the work were appropriately investigated and resolved.

\section{Ethics approval and consent to participate}

Investigators obtained local ethics committee approval of the protocol, which was registered at ClinicalTrials.gov (NCT02686034). All patients provided written informed consent in advance of study participation.

\section{Consent for publication}

Not applicable.

\section{Competing interests}

P. Martelletti has received research grants, advisory board fees, or travel fees from ACRAF; Allergan S.p.A.; Amgen Inc.; electroCore, Inc.; Novartis AG; and Teva Pharmaceutical Industries Ltd.

L. Grazzi has received consultancy and advisory fees from Allergan S.p.A. and electroCore, Inc

G. Pierangeli has nothing to disclose.

I. Rainero has received consultancy fees from electroCore, Inc., and Mylan N.V. and research grants from the European Commission - Horizon 2020. He is also a principal investigator for RCTs sponsored by Axovant Sciences Ltd. and TauRx Pharmaceuticals Ltd.

P. Geppetti has received consultancy fees from Allergan S.p.A.; electroCore, Inc.; Evidera; Novartis AG; Pfizer Inc.; and Sanofi S.p.A. and research grants from Chiesi Farmaceutici S.p.A. He is also a principal investigator for RCTs sponsored by Eli Lilly and Company; Novartis AG; and Teva Pharmaceutical Industries Ltd.

A. Ambrosini has received consultancy fees from Almirall, S.A., and travel grants from Allergan S.p.A., Almirall, S.A., and Novartis.

P. Sarchielli has received clinical study fees from Allergan S.p.A.

P. Barbanti has received consultancy fees from Allergan S.p.A.; electroCore, Inc.; Janssen Pharmaceuticals, Inc.; Lusofarmaco; and Visufarma and advisory fees from Abbott Laboratories; Merck \& Co., Inc.; Novartis AG; and Teva Pharmaceutical Industries Ltd. He is also a principal investigator for RCTs sponsored by Alder BioPharmaceuticals 
Inc.; Eli Lilly and Company; GlaxoSmithKline Pharmaceuticals Ltd.; and Teva Pharmaceutical Industries Ltd.

C. Tassorelli has consulted for Allergan S.p.A.; electroCore, Inc.; Eli Lilly and Company; and Novartis AG and has received research grants from the European Commission and the Italian Ministry of Health. She is also a principal investigator or collaborator for RCTs sponsored by Alder BioPharmaceuticals Inc.; Eli Lilly and Company; Novartis; and Teva Pharmaceutical Industries Ltd.

E. Liebler is an employee of electroCore, Inc., and receives stock ownership. M. de Tommaso has received advisory fees from Allergan S.p.A.; Neopharmed; and Pfizer Inc.

\section{Publisher's Note}

Springer Nature remains neutral with regard to jurisdictional claims in published maps and institutional affiliations.

\section{Author details}

'Department of Clinical and Molecular Medicine, Sapienza University, Rome, Italy. ${ }^{2}$ Headache and Pain Unit, Istituto di Ricovero e Cura a Carattere Scientifico (IRCCS) San Raffaele Pisana, Rome, Italy. ${ }^{3}$ Neuroalgology Unit, Carlo Besta Neurological Institute and Foundation, Milan, Italy. ${ }^{4}$ RCCS Istituto delle Scienze Neurologiche di Bologna, Bologna, Italy. ${ }^{5}$ Department of Neuroscience, University of Turin, Turin, Italy. ${ }^{6}$ Headache Centre, University Hospital of Careggi, Florence, Italy. ${ }^{7}$ RCCS Neuromed, Pozzilli, IS, Italy. ${ }^{8}$ Neurologic Clinic, Santa Maria della Misericordia Hospital, Perugia, Italy. ${ }^{9}$ Headache Science Centre, IRCCS C. Mondino Foundation, Pavia, Italy. ${ }^{10}$ Department of Brain and Behavioral Sciences, University of Pavia, Pavia, Italy. ${ }^{11}$ electroCore, Inc., Basking Ridge, NJ, USA. ${ }^{12}$ Neurophysiology and Pain Unit, University of Bari Aldo Moro, Bari, Italy.

\section{Received: 9 August 2018 Accepted: 3 October 2018}

Published online: 01 November 2018

\section{References}

1. Lopes M, Dunn JD, Calhoun AH, Rapoport AM (2012) Concepts in acute migraine management: clinical and managed care perspectives. Am J Pharm Benefits 4(5):201-206

2. Negro A, Koverech A, Martelletti P (2018) Serotonin receptor agonists in the acute treatment of migraine: a review on their therapeutic potential. J Pain Res 11:515-526. https://doi.org/10.2147/JPR.S132833

3. Puledda F, Messina R, Goadsby PJ (2017) An update on migraine: current understanding and future directions. J Neurol 264(9):2031-2039. https://doi. org/10.1007/s00415-017-8434-y

4. Rolan PE (2012) Drug interactions with triptans: which are clinically significant? CNS Drugs 26(11):949-957. https://doi.org/10.1007/s40263-012-0002-5

5. Bigal ME, Lipton RB (2009) Overuse of acute migraine medications and migraine chronification. Curr Pain Headache Rep 13(4):301-307

6. Bonafede M, Sapra S, Shah N, Tepper S, Cappell K, Desai P (2018) Direct and indirect healthcare resource utilization and costs among migraine patients in the United States. Headache 58(5):700-714. https://doi.org/10.1111/head.13275

7. Friedman BW, Irizarry E, Solorzano C, Latev A, Rosa K, Zias E, Vinson DR, Bijur PE, Gallagher EJ (2017) Randomized study of IV prochlorperazine plus diphenhydramine vs IV hydromorphone for migraine. Neurology 89(20): 2075-2082. https://doi.org/10.1212/WNL.0000000000004642

8. Friedman BW, West J, Vinson DR, Minen MT, Restivo A, Gallagher EJ (2015) Current management of migraine in US emergency departments: an analysis of the National Hospital Ambulatory Medical Care Survey. Cephalalgia 35(4):301-309. https://doi.org/10.1177/0333102414539055

9. Orr SL, Friedman BW, Christie S, Minen MT, Bamford C, Kelley NE, Tepper D (2016) Management of Adults with Acute Migraine in the emergency department: the American headache society evidence assessment of parenteral pharmacotherapies. Headache 56(6):911-940. https://doi.org/10.1111/head.12835

10. Lipton RB, Dodick DW, Silberstein SD, Saper JR, Aurora SK, Pearlman SH, Fischell RE, Ruppel PL, Goadsby PJ (2010) Single-pulse transcranial magnetic stimulation for acute treatment of migraine with aura: a randomised, double-blind, parallel-group, sham-controlled trial. Lancet Neurol 9(4):373380. https://doi.org/10.1016/S1474-4422(10)70054-5

11. Tassorelli C, Grazzi L, de Tommaso M, et al. (2018) Non-invasive vagus nerve stimulation as acute therapy for migraine: the randomized PRESTO study. Neurology 91(4):e364-e373.
12. Barbanti P, Grazzi L, Egeo G, Padovan AM, Liebler E, Bussone G (2015) Noninvasive vagus nerve stimulation for acute treatment of high-frequency and chronic migraine: an open-label study. J Headache Pain 16:61. https://doi. org/10.1186/s10194-015-0542-4

13. Goadsby PJ, Grosberg BM, Mauskop A, Cady R, Simmons KA (2014) Effect of noninvasive vagus nerve stimulation on acute migraine: an open-label pilot study. Cephalalgia 34(12):986-993. https://doi.org/10.1177/0333102414524494

14. Kinfe TM, Pintea B, Muhammad S, Zaremba S, Roeske S, Simon BJ, Vatter H (2015) Cervical non-invasive vagus nerve stimulation (nVNS) for preventive and acute treatment of episodic and chronic migraine and migraineassociated sleep disturbance: a prospective observational cohort study. J Headache Pain 16:101. https://doi.org/10.1186/s10194-015-0582-9

15. Silberstein SD, Calhoun AH, Lipton RB, Grosberg BM, Cady RK, Dorlas S, Simmons KA, Mullin C, Liebler EJ, Goadsby PJ, Saper JR, Group ES (2016) Chronic migraine headache prevention with noninvasive vagus nerve stimulation: the EVENT study. Neurology 87(5):529-538. https://doi.org/10. 1212/WNL.0000000000002918

16. (2013) The International Classification of Headache Disorders, 3rd edition (beta version). Cephalalgia 33(9):629-808. https://doi.org/10.1177/0333102413485658

17. Akerman S, Simon B, Romero-Reyes M (2017) Vagus nerve stimulation suppresses acute noxious activation of trigeminocervical neurons in animal models of primary headache. Neurobiol Dis 102:96-104. https://doi.org/10. 1016/j.nbd.2017.03.004

18. Hawkins $\mathrm{JL}$, Cornelison LE, Blankenship BA, Durham PL (2017) Vagus nerve stimulation inhibits trigeminal nociception in a rodent model of episodic migraine. Pain Rep 2(6):e628. https://doi.org/10.1097/PR9.0000000000000628

19. Oshinsky ML, Murphy AL, Hekierski H Jr, Cooper M, Simon BJ (2014) Noninvasive vagus nerve stimulation as treatment for trigeminal allodynia. Pain 155(5):1037-1042. https://doi.org/10.1016/j.pain.2014.02.009

20. Ferrari MD, Roon KI, Lipton RB, Goadsby PJ (2001) Oral triptans (serotonin 5$H T(1 B / 1 D)$ agonists) in acute migraine treatment: a meta-analysis of 53 trials. Lancet 358(9294):1668-1675. https://doi.org/10.1016/S0140-6736(01)06711-3

21. Cady R, Elkind A, Goldstein J, Keywood C (2004) Randomized, placebocontrolled comparison of early use of frovatriptan in a migraine attack versus dosing after the headache has become moderate or severe. Curr Med Res Opin 20(9):1465-1472. https://doi.org/10.1185/030079904X2745

22. Grazzi L, Tassorelli C, de Tommaso M, Pierangeli G, Martelletti P, Rainero I, Geppetti P, Ambrosini A, Sarchielli P, Liebler E, Barbanti P (2018) Practical and clinical utility of non-invasive vagus nerve stimulation (nVNS) for the acute treatment of migraine: post hoc assessment of the randomized, sham-controlled, double-blind PRESTO trial [abstract MTIS2018-059]. Cephalalgia 38(1S):42. https://doi.org/10.1177/0333102418789865

23. Cady RK, Sheftell F, Lipton RB, O'Quinn S, Jones M, Putnam DG, Crisp A, Metz A, McNeal S (2000) Effect of early intervention with sumatriptan on migraine pain: retrospective analyses of data from three clinical trials. Clin Ther 22(9):1035-1048

24. Gaul C, Diener HC, Silver N, Magis D, Reuter U, Andersson A, Liebler El, Straube A, Group PS (2016) Non-invasive vagus nerve stimulation for prevention and acute treatment of chronic cluster headache (PREVA): a randomised controlled study. Cephalalgia 36(6):534-546. https://doi.org/10.1177/0333102415607070

25. Goadsby PJ, de Coo IF, Silver N, Tyagi A, Ahmed F, Gaul C, Jensen RH, Diener HC, Rabe K, Straube A, Liebler E, Marin J, Ferrari MD, Group AS (2018) Non-invasive vagus nerve stimulation for the acute treatment of episodic and chronic cluster headache: findings from the randomized, double-blind, sham-controlled ACT2 study. Cephalalgia 38(5):959-969. https://doi.org/10.1177/03331024177444362

26. Silberstein SD, Mechtler LL, Kudrow DB, Calhoun AH, McClure C, Saper JR, Liebler EJ, Rubenstein Engel E, Tepper SJ, Group AS (2016) Non-invasive vagus nerve stimulation for the acute treatment of cluster headache: findings from the randomized, double-blind, sham-controlled ACT1 study. Headache 56(8): 1317-1332. https://doi.org/10.1111/head.12896

27. Asano E, Goadsby PJ (2013) How do we fashion better trials for neurostimulator studies in migraine? Neurology 80(8):694. https://doi.org/10. 1212/WNL.0b013e3182825174

28. Moeller M, Schroeder CF, May A (2018) Comparison of active and "sham" non-invasive vagal nerve stimulation on lacrimation in healthy volunteers [abstract PS76]. Headache 58(suppl 2):135-136. https://doi. org/10.1111/head. 13306 